\section{Questión}

Periodismo / Comunicación

ISSN 1669-6581

La comunicación del patrimonio universitario

Ailén Fonseca Martínez, Maribel Brull González, Ana María Guerra Casanellas

Question/Cuestión, Nro.66, Vol.2, agosto 2020

ISSN: 1669-6581

https://perio.un/p.edu.ar/ojs/index.php/question/

IICom - FPyCS - UNLP.

DOI: https//doi.org/10.24215/16696581e491

\title{
La comunicación del patrimonio universitario
}

\section{The communication of university heritage}

\section{Ailén Fonseca Martínez}

ailen.fonseca@uo.edu.cu

https://orcid.org/0000-0002-9079-1525

Docente, profesora instructora de la Facultad de Humanidades, Universidad de

Oriente, Cuba.

\section{Maribel Brull González}

brull@uo.edu.cu

https://orcid.org/0000-0001-6253-9915

Doctora en Ciencias de la Comunicación. Profesora Titular. Máster en Comunicación. Vicedecana de Investigaciones y Postgrado; Vicepresidenta del Consejo Científico de la Facultad de Humanidades y electa como Miembro del Consejo Científco de la Universidad de Oriente. Miembro del Tribunal Nacional de Grados Científicos en Ciencias de la Información y Ciencias de la 
Comunicación de la Universidad de La Habana. Miembro de la Junta de Acreditación Nacional (JAN) para la evaluación de programas de pregrado y postgrados e instituciones educativas. Miembro del Consejo provincial de

Ciencias Sociales en Santiago de Cuba. Posee titulación de Gerente Comercial. Graduada en cursos de Protocolo y Eventos. Diplomada en Redacción Publicitaria. Diplomada en Relaciones Internacionales Universitarias. Licenciada en Filosofía e Historia. Premio Nacional de Comunicación en la Especialidad de Relaciones Públicas.

\section{Ana María Guerra Casanellas}

casanellc@up.edu.cu

https://orcid.org/0000-0001-8392-4134

Doctora en Ciencias Lingüísticas (2007). Profesora Titular de la carrera de Letras en la Universidad de Oriente. Miembro de la Comisión Nacional de Carrera. Ha formado parte de varios Proyectos de Investigación. Coordinadora del programa de Maestría Estudios de Lengua y Discursos. Miembro de

claustro de maestrías de otras facultades en la UO Maestría en Desarrollo Cultural Comunitario, Maestría en Comunicación y maestría en lengua inglesa). Tiene varias publicaciones y presentaciones en congresos. Ha dirigido tesis de maestría y de doctorado. Miembro del Comité Académico del Doctorado en Ciencias Lingüísticas y Literarias de la Universitario.

\section{Resumen}

La gestión del patrimonio se ha incorporado de forma creciente al lenguaje de las universidades, enriqueciéndose con nuevas prácticas culturales y científicas relacionadas con los conceptos de salvaguarda y puesta en valor. Es por ello 
que este artículo propone una aproximación a la comunicación del patrimonio universitario como tarea investigativa necesaria para alcanzar inclusiones más dinámicas e integrales de la producción misma del sentido social, a partir de las disposiciones internacionales y en un espacio de confluencia con las Tecnologías de la Información y la Comunicación (TIC).

\title{
Palabras clave
}

Gestión, Comunicación, Patrimonio Universitario

\begin{abstract}
Heritage management has been increasingly incorporated into the language of universities, eriching itself with new cultural and scientific practices related to safeguarding and enhancing value.That is why this article proposes an approach to the communication of the university heritage as a necessary investigative task to achieve more dynamic and comprehensive inclusions of the production of the social sense itself, based on international provisions and in a space of confluence with Technologies Information and Communication (TIC).
\end{abstract}

\section{Key words}

Management, Communication, University Heritage.

\section{Introducción}

El proceso de comunicación contribuye a reforzar la significación comunitaria y social del patrimonio. Por esta razón, se aborda como una cuestión de vital importancia en la activación y valoración patrimonial. Su planificación permite 
un mejoramiento en las relaciones de las instituciones e investigadores que trabajan el tema con el objetivo de una mayor visibilidad ante la sociedad.

Actualmente los centros de estudios superiores ocupan espacios de reflexión y acción cada vez mejor orientados hacia la identificación, reconocimiento y comunicación de su patrimonio. Se enfrentan a la formación e investigación en el contexto de un nuevo mundo urgido de conocimientos actualizados en correspondencia con el desarrollo de la ciencia, la tecnología y la innovación basado en la restauración y conservación de la identidad.

$Y$ es que se han convertido en reservorios de bienes materiales e inmateriales que dan fe de los continuos procesos históricos, académicos, científicos, extensionistas y culturales que allí acontecen. Inmuebles excepcionales por su arquitectura, bienes muebles de diversa naturaleza: artísticos, científicos, numismáticos, documentales, monumentos y museos, costumbres, ritos y tradiciones, componen, entre otros, la diversidad de valores atesorados.

\section{Comunicación del patrimonio}

La gestión del patrimonio tiene en la comunicación uno de los elementos por el que deberá apostar más en los próximos años para contribuir a la socialización; un potencial sobre el que autores como Campillo (1998) y Mejías (2008) han reflexionado en sus investigaciones. Querol y Martínez (1999) al referirse a acciones para proteger y conservar el patrimonio afirman que " nada tendría sentido si no se establecieran comunicaciones entre el patrimonio y la propia sociedad" (p. 28), porque la atención a la diversidad de los individuos comienza con la comunicación del patrimonio, como forma de mediación entre el patrimonio y las personas. 
Desde esta perspectiva ResiK (2017) y Dávila (2018) destacan que la comunicación del patrimonio no es un tema de larga data en el campo académico. Señalan además que los procesos comunicativos se han relacionado más al entramado de complejos y prácticas culturales y no a las especificidades de la materia patrimonial con fines estratégicos para el desarrollo y el cambio social, aún cuando consideran que es impensable un ejercicio de acercamiento a valores patrimoniales sin el protagonismo de la comunicación.

Otros autores como Ballart y Tresserras (2001), García (2006) y Gómez (2006) hablan de comunicación del patrimonio de manera general para englobar varias disciplinas que se relacionan entre sí: difusión del patrimonio, educación del patrimonio e interpretación del patrimonio. Pero que a pesar de su heterogeneidad se considera, tendrían en común dos principios básicos:

a) Un poderoso compromiso implícito y un conjunto común de actitudes dirigidas a proteger y conservar para la posteridad los objetos, lugares naturales, mo-numentos, estructuras y otras evidencias materiales del pasado y presente de una comunidad dada.

b) Una percepción compartida del valor intrínseco y cultural del patrimonio y el propósito de usarlo para comunicar e interpretar el pasado para las generaciones tanto presentes como futuras.'

Al hablar de comunicación del patrimonio cultural, Valdés (1999) la define como:

Las diversas formas que puede asumir la transmisión y/o representación de bienes y obras culturales, creando las condiciones para que los posibles usuarios entren en contacto con el bien o servicio cultural 
deseado, que implica una función de aproximar el patrimonio cultural a la población (p.2).

Es Mateos (2008) quien con mayor flexibilidad define el término, al incluir en una misma conceptualización dos ideas fundamentales que hasta entonces habían sido tratadas por separado:

1. la difusión cultural, que busca la plena accesibilidad intelectual y emocional del patrimonio mediante el uso de disciplinas como la interpretación y la didáctica, y que incluye a su vez estrategias de difusión como la difusión preventiva.

2. y la de ejecución de la comunicación, la cual puede ser comercial, es decir tiene la finalidad de atraer y fidelizar al público destinatario para que este conozca y visite el recurso patrimonial, o corporativa, cuyo objetivo es crear, gestionar, modificar y mantener la identidad corporativa de la organización pública o privada que lidera un determinado proyecto patrimonial.

Su definición de comunicación global del patrimonio se podría decir que contiene un modelo de gestión sostenible a partir de cinsiderarla como la gestión cultural y comunicativa que media entre los recursos patrimoniales y la sociedad para potenciar un uso responsable, provechoso, atractivo y efectivo capaz de aunar la preservación de los bienes culturales con su disfrute integral por parte de los usuarios (Mateos, 2008).

Se comprende entonces que esta será la forma para conectar de manera bidireccional al patrimonio con la sociedad. Según Fontal, "el principal objetivo de la comunicación global del patrimonio será siempre dar a conocer los aspectos relacionados con el bien patrimonial” (2008, p.89). Es decir, que el 
elemento prioritario, es el patrimonio, la valoración y conservación son concebidos como efectos colaterales de la comunicación.

\section{Comuniación del patrimonio universitario}

A partir del reconocimiento de la importancia de su producción material y espiritual, los centros universitarios se enfrentan a la urgente necesidad de adquirir y fomentar el respeto y conocimiento de lo auténtico; no para desdeñar lo diferente, sino para distinguir lo que es propio de lo ajeno, lo que es natural de lo que resulta impuesto. Este fenómeno ocurre a una velocidad que en ocasiones no permite asimilarlo ni evaluarlo, lo cual lleva a la sociedad a una enajenación de su propia realidad y a un constante enfrentamiento valorativo entre su bagaje cultural y los elementos que la modernidad le impone, apoyándose en la destrucción y menoscabo de la obra y la singularidad de los pueblos.

La reflexión y el debate se centran hoy en diferentes espacios, no solo desde el ámbito del mercado cultural, sino desde los escenarios de comercialización, en el cual está inmersa la globalización económica, la industria del entretenimiento y el accionar mediático. La globalización cultural es un efecto creciente, hace que el mundo esté interconectado desde los más diversos terrenos, económico, social, tecnológico, político y especialmente en áreas ciberculturales. Se han incrementado las posibilidades de encuentros multiculturales, los cuales favorecen la comunicación simultánea de actividades generadas por diferentes redes masivas que propician mensajes, productos y bienes simbólicos ajenos.

Este asunto emerge en la polémica cuando para muchos irradia desde lo positivo, al compartir costumbres, tradiciones y alianzas entre mercados; mientras que para otros es intentar desde lo transnacional socavar, las raíces 
de las pueblos, condicionando a ciudadanos o cibernautas a generar una opinión pública de poder, sustentados en patrones culturales que se basan en la manipulación mediante la propaganda, la publicidad o las formas de ejercer las relaciones públicas, logrando silenciar o neutralizar identidades, en ocasiones mediante el uso de discursos arrogantes y autoritarios o con el montaje de espectáculos que se sirven de mensajes escogidos para redimensionar el comportamiento de grandes masas.

Preparar a las futuras generaciones para entender su relación con el patrimonio más allá de toda consideración formal es un deber, que transita por la épica que se ha consustanciado en su representación. Las instituciones de enseñanza superior en su dimensión renovadora constituyen un importante espacio para el intercambio más provechoso, por su aporte al conocimiento, al desarrollo y al diálogo cultural; en sí mismas, argumentan la necesidad de ser protagonistas en la protección de su patrimonio. Se convierten en agentes custodios de esas huellas del pasado y por lo tanto son realidades vivas que facilitan la interpretación y comprensión de su devenir, donde la identidad es el producto social para poderse identificar.

En múltiples ocasiones una de las causas fundamentales en el tratamiento inadecuado al patrimonio y el desinterés de las comunidades universitarias por su preservación lo constituye el desconocimiento de los valores patrimoniales, porque la pertenencia y la identidad se fortalecen en la medida que los hombres puedan ser capaceces de reconocerse en su entramado significativo. La participación de los universitarios en la vida social deviene una oportunidad para lograr la unidad entre lo diverso, el registro de la identidad, el desarrollo, la cultura científica y la extensión del saber. 
A través de esta dinámica continua se ha transitado gradualmente hacia el pleno reconocimiento de la verdadera amplitud del patrimonio de las casas de altos estudios y la lucha por la trascendencia de buscar permanentemente su preservación. Así, la disyuntiva en cuanto a producir conocimiento desde nuevos paradigmas, ha devenido en el cuestionamiento a la concepción tradicional de la gestión del conocimiento, para puntualizar alternativas más novedosas y flexibles, cuyo fundamento se enraíza en lo interno y la transdisciplinariedad como cimiento epistemológico.

Se trata entonces de preparar a los universitarios con las herramientas que le permitan la comprensión de cada bien de interés cultural que les rodea, incluidos aquellos símbolos del patrimonio inmaterial, de los cuales son portadores activos. En tal sentido "para lograr los objetivos comunicacionales en el contexto del patrimonio, será imprescindible que la comunicación sea blanca, veraz, positiva y no agresiva" (Mateos, 2009, p.7).

Además deberán ajustarse siempre las expectativas generadas con las acciones comunicativas pertinentes a la experiencia real del usuario, pues son los saberes y las tradiciones populares ubicados en las cotidianidades los que se colocan jerárquicamente en las expresiones más recurrentes de la identidad a nivel de la comunidad. Su significación, lectura e interpretación constituyen en la actualidad uno de los elementos más importantes para comprender la vigencia del pasado en el presente porque "ningún proyecto de desarrollo tendrá éxito mientras los destinatarios no se apropien de él”. (López, 2000, p.312).

Desde una conexión muy cercana con la educación patrimonial, la comunicación, facilita las relaciones que se establecen entre patrimonio, sociedad e instituciones culturales/educativas mediante la teoría de la 
comunicación y siempre en relación con los procesos de enseñanza y aprendizaje (Cristofano y Palazzetti, 2011; Mateos, 2008). De acuerdo con el punto de vista de Martín y Cuenca (2015), el patrimonio no es posible sin el proceso de comunicación y sin comunicación, no se puede desarrollar el proceso educativo.

Por eso idealmente, la comunicación del patrimonio universitario debe ser bidireccional, estratégica, comprensible, emotiva y experiencial. Siempre debe transmitir unos valores asociados al patrimonio que sean significativos para el receptor, y además de concienciar sobre la necesidad de conservación, fomentar la participación de los públicos y buscar su apoyo en la realización de actividades.

El interés público en temas referentes al patrimonio puede estar determinado por la disponibilidad y la divulgación de la información que se promuevan, es decir que "la mera existencia del activo patrimonial o la intervención en el mismo no despiertan interés social, sino que este debe ser trabajado desde estrategias de comunicación bien dirigidas" (Escalante y Huiltrón, 2017, p.139). Para comunicar el patrimonio también se tendrán en cuenta "la adecuación y la eficacia, y como en cualquier tipo de comunicación, será fundamental experimentar y arriesgarse para diferenciarse y resultar memorable" (Díaz, 2008 , p. 176). Es preciso entonces, un proyecto que aúne la investigación, la elaboración de conocimientos y la valoración del patrimonio, y una coherente labor de conservación que permita la interpretación y presentación.

A su vez, los nuevos escenarios ofrecen oportunidades diversas para articular propuestas que incluyan el trabajo interdisciplinario; "la comunicación global sienta las bases para establecer sinergias interdisciplinarias entre ámbitos de estudio que no se encuentran, como los de la ciencia pura con las disciplinas 
humanísticas, dentro de los cuales se analizan los temas del valor del patrimonio" (Niglio, 2013, p.26). De acuerdo con Hermon y Niccolucci (2007) "otra de sus peculiaridades es que no debe perder de vista la interdisciplinariedad característica del objeto a comunicar" (2007, p.2), por lo que se recomiendan los acercamientos holísticos.

A la comunicación adecuada se refiere Capriotti (2008) desde la difusión de la identidad y la diferencia del patrimonio: "Si no se comunica su identidad, [...] si no se comunica su diferencia, [...] aunque el patrimonio exista los públicos no lo conocerán. Aunque sea diferente los públicos no lo sabrán” ( p.133). Y por comunicación eficaz se entiende a "aquella diseñada para la situación, tiempo, espacio y audiencia” (Cutlip, Center \& Broom, 1994, p. 349).

De esta forma se puede afirmar que para comunicar el patrimonio universitario es preciso entenderlo como un elemento del acervo cultural, natural o histórico. No se trata por tanto de venerar los valores patrimoniales, sino de conocerlos y conservarlos para el enriquecimiento personal y comunitario, ya que "la custodia del pasado encuentra su verdadero sentido cuando se convierte en animador de progreso presente y en constructor de futuro" (Aznar, 2006, p. 13). Así historia y memoria articulan relatos que dotan de sentido al pasado construido sobre los bienes culturales, los hacen significativos y les otorgan un valor patrimonial que sin esas miradas serán objetos inertes.

En tal sentido, Escalante y Huiltrón (2017), inspirados en perspectivas como la de García (1999) y Martínez (2016) afirman que para preservar el patrimonio se debe aspirar a una apropiación colectiva y democrática del mismo, donde es necesaria la creación de condiciones materiales y simbólicas disponibles para el público, con el objetivo de que este lo encuentre significativo y lo comparta. 


\section{Accionar de las organizaciones internacionales en la comunicación del patrimonio universitario}

La Organización de las Naciones Unidas para la Educación, la Ciencia y la Cultura (UNESCO) ha incluido tradicionalmente en sus disposiciones legales, normas y textos doctrinales que señalan la importancia de la comunicación para contribuir a preservar el patrimonio de las naciones y del mundo. Declaraciones, cartas, convenciones y hasta en la Constitución de la Organización aprobada el 16 de noviembre de 1945 se hace alusión a la comunicación como herramienta significativa para la protección y la puesta en valor.

De esta forma en 1972 la Convención para la Protección del Patrimonio Mundial, Cultural y Natural hace un llamado a la cooperación para la conservación, el progreso y la difusión del saber del patrimonio universal. Así, se contrajo por los Estados Partes en el Artículo 27 la obligación de informar al público por todos los medios sobre las amenazas del patrimonio natural y cultural.

A tales efectos, el Fondo de Patrimonial Mundial creado también en 1972 concibe entre la líneas de investigación para la asistencia internacional el apoyo a la educación y a la promoción en dos líneas de trabajo: información destinada al público en general o para grupos de intereses específicos y el desarrollo de programas de enseñanza y material educativo para su utilización en escuelas, colegios y universidades.

La divulgación del concepto de Patrimonio Mundial, a través de estrategias de comunicación, educación, investigación, formación de capacidades y concienciación y la recomendación de aumentar a través de la comunicación, los niveles de participación y apoyo en las actividades vinculadas a este tema, 
fueron propuestas de la Declaración de Budapest, durante la Vgésimo Sexta Sesión del Comité de Patrimonio Mundial, en 2002.

En el año 2003 cuando se reubricó la Convención para la Salvaguarda del Patrimonio Cultural Inmaterial, en el artículo 14 se declaran recomendaciones para la creación de programas educativos y de difusión de la información dirigidos al público específicamente joven.

Ratificada por la Décimo Sexta Asamblea General del Consejo Internacional de Sitios y Monumentos (ICOMOS), en octubre de 2008 la Carta para la Intertpretación y Presentación de Sitios del Patrimonio Cultural establece las líneas profesionales para los desafíos específicos de la conservación e impulsa la comunicación eficaz sobre el patrimonio en cada región del mundo. Aquí se destaca además la importancia de la comunicación pública como parte primordial en un proceso de conservación más amplio describiéndolo como difusión, divulgación, presentación e interpretación; y se reconoce de forma implícita que cada acto de conservación de patrimonio dentro de todas las tradiciones culturales del mundo es por su naturaleza un acto comunicativo.

A escala internacional desde inicios del siglo XXI son abundantes las experiencias de gestión asociadas al patrimonio universitario con altos niveles de protección y difusión. La creciente concientización de sus particularidades e importancia generan sucesivos balances críticos que han conducido a transformaciones y actualizaciones en las estrategias para su cuidado, además de una consolidación conceptual que se aprecia en publicaciones y foros especializados. Sin embargo, son insuficientes las aproximaciones a los propios límites y particularidades de lo considerado patrimonio universitario al relacionarlo solo con los elementos que lo conforman en sí, y no con el valor que le otorgan las personas que lo identifican como tal. 
En el año 2000 se formalizó la Red Europea de Patrimonio Académico (UNIVERSEUM), de cuyos encuentros se destacan textos como Arranging and rearranging: planning university heritage for the future (2012) y Shaping European university heritage past and possible future (2013), de donde emana un acercamiento de la comunidad académica al valor patrimonial asociado a las universidades.

La Asamblea General del Consejo Internacional de Museos (ICOM) que tuvo lugar en Barcelona en julio de 2001 fue el escenario para la creación del Comité Internacional de Museos y Colecciones Universitarias (UMAC); de esta forma se dotaba así al patrimonio universitario de una identidad diferenciada en el ámbito de los museos.

El propósito de estos primeros, pero importantes pasos en la gestión del patrimonio de los centros de educación superior es destacar el enfoque del patrimonio cultural como un actor de responsabilidad colectiva, garante de valores morales, intelectuales y humanos.

En el texto normativo de alcance internacional dedicado al patrimonio cultural universitario la Recomendación (2005), derivado del Consejo de Europa se presentan algunas manifestaciones que adopta el patrimonio universitario, y se apunta constantemente a la composición por bienes materiales e inmateriales. De esta forma refiere que algunos de los elementos constitutivos del patrimonio universitario serían:

- El patrimonio universitario integra a todo el patrimonio material e inmaterial relacionado a las instituciones, organismos y sistemas de la educación superior, así como de la comunidad universitaria de profesores y estudiantes, y al entorno social y cultural del cual forma parte este patrimonio. 
- El patrimonio universitario debe ser entendido como todas las huellas de la actividad humana relacionada con la educación superior.

- Es una fuente acumulativa de riquezas en relación directa con la comunidad académica de profesores y estudiantes, sus creencias, valores, logros y su rol social y función cultural en tanto maneras de transmisión de conocimiento y capacidad de invasión.

Otro documento que se destaca es la Declaración de Alcalá sobre la protección, conservación y difusión del patrimonio universitario; en él se apuesta por la comprensión de que con la evolución de las sociedades, las universidades se han convertido en un importante caudal de conocimientos para el desarrollo universal, y que el conjunto de recintos históricos se convierte en testigo excepcional de este hecho para las futuras generaciones (Rivera, 2013). Aquí se apunta a la condición verdaderamente universal de los centros de educación superior y sus valores intrínsecos.

\section{Las TIC en la comunicación del patrimonio universitario}

La comunicación del patrimonio como producto social, está expuesta también a los cambios impuestos por el desarrollo tecnológico, que no solo incrementa las herramientas comunicativas, sino que ha transformado las estructuras más tradicionales, lo que genera procesos interactivos novedosos. El diseño de estrategias basadas en el uso de las Tecnologías de la Información y las Comunicaciones (TIC) constituye una oportunidad para dar a conocer y hacer accesibles los valores patrimoniales. La expansión de herramientas tecnológicas que permiten un mayor alcance a la información y conocimiento del patrimonio han favorecido un uso más amplio a través de las redes virtuales en las universidades. 
Para realizar una aproximación a la comuniación patrimonial universitaria es necesario entender entonces el nuevo contexto en el que, tanto los públicos como los elementos culturales se encuentran inmersos. De acuerdo con Castells (2009), en este contexto sociocultural se requiere formas de organización más proactivas, interconectadas y orientadas al funcionamiento en red, pues las nuevas plataformas han venido no solo a multiplicar las posibilidades de emisión de los mensajes, también han provocado la incorporación de nuevos formatos de comunicación. Mateos (2012) explica que los activos patrimoniales son potentes instrumentos comunicativos, que para llegar a sus seguidores necesitan además de los medios tradicionales, aquellos que usan las nuevas fórmulas generadas por las TIC.

En el ámbito concreto del patrimonio universitario las TIC constituyen un apoyo fundamental en su presentación e interpretación. Desde esa perspectiva Mejías (2008) afirma que una de las oportunidades del patrimonio cultural es precisamente "la gran potencialidad de crecimiento en el campo de la divulgación y comunicación del patrimonio y la incorporación estratégica de nuevas tecnologías de la información y comunicación a la gestión del patrimonio histórico" (p.19).

Las nuevas capacidades de conexión en red que proporcionan las TIC posibilitan que el público pueda producir, revisar, proponer y criticar a través de plataformas participativas donde el propio conocimiento que se genera estimula la creación de nuevas aplicaciones y servicios. Las acciones digitales con el trancurso del tiempo responden menos a la intuición y se profesionalizan de forma progresiva con estudios de público, análisis de redes para justificar la creación de perfiles, planificación de objetivos, categorización y periodicidad de 
contenidos; "cada una de ellas es capaz de servir a distintos propósitos" (Mateos, 2009, p.12).

Hoy se puede afirmar que la definición de audiencia activa ha sustituido al de audiencia pasiva. En tal sentido Mendes (2002) muestra la relación entre el ciberespacio y la interactividad, además señala su importancia para el conocimiento y apropiación del patrimonio construido:

El ciberespacio modifica la forma en que las personas entienden el patrimonio edificado y se relacionan con él [...] Es fundamentalmente un objeto de conocimiento que puede ser utilizado en función de los objetivos de numerosos y diversos proyectos intelectuales. Además, se trata de un objeto operacional, con el que se puede llevar a cabo cualquier tipo de experimento y someter a pruebas teorías, significados y sensaciones, así como establecer relaciones entre los idividuos y otros objetos (p.25).

Esta forma de comprender al receptor ha dejado atrás como señala Torres (2012), "los términos como público general o audiencia de masa para ir segmentando a las audiencias, ofreciendo productos diferenciados para cada grupo de personas", (p.65); es decir una forma de comunicación más interactiva.

La incorporación de las TIC a la comunicación del patrimonio universitario es una cuestión de análisis imprescindible también a la hora de abordar la construcción de nuevos modelos de enseñanza-aprendizaje, en el que se debe considerar el potencial educativo expuesto, así como su rápida integración en las dinámicas comunicativas. Con el propósito de mostrar cómo su desarrollo ha permitido ampliar las posibilidades de participación y las formas, formatos, medios y soportes para comunicar en el campo patrimonial de los públicos, "el 
avance en las investigaciones sobre nuevas tecnologías aplicadas al campo patrimonial ha tenido una gran acogida permitiendo obtener mejores resultados en lo que se refiere al conocimiento y difusión de los contenidos" (Ruiz, 2011, p.1).

Otros autores como Irala (2013) expresan la relación abierta que se establece entre patrimonio, difusión y TIC:

Las nuevas tecnologías son fundamentales para la eficaz gestión y puesta en valor del Patrimonio Histórico-Artístico. Tanto en la fase de catalogación como en la difusión pública de los fondos las TIC aportan fórmulas para la eficiencia de nuestro trabajo y la conservación futura de nuestro valioso patrimonio (p.169).

Varias son las experiencias que involucran la comunicación del patrimonio y las nuevas tecnologías. Existe una importante y creciente tendencia a su empleo en la gestión de todos los procesos patrimoniales, que van desde el diagnóstico hasta la puesta en valor. Los sitios web corporativos, mediante los que se da a conocer la información institucional se han convertido en relevantes alternativas para potenciar y realizar visitas y recorridos virtuales. En las publicaciones electrónicas se presentan las revistas especializadas, en las que se articula la investigación con la difusión de resultados.

A nivel internacional se destacan importantes estudios, dentro de los que se encuentran, de Fagundes (2010), Comunicación, cultura y sociedad digital en Brasil, en él se analizan de manera crítica las transformaciones culturales de la sociedad brasileña y se relacionan aspectos significativos en este contexto cultural latinoamericano de habla portuguesa como las tensiones entre territaorialidades y estado nacional, y entre diversidad, identidad y patrimonio cultural. 
El documental interactivo como instrumento educativo. Propuesta teórica práctica para la difusión del patrimonio cultural colombiano de Arciniegas (2016), propone el documental interactivo como instrumento educativo para la difusión del patrimonio cultural; y tras el análisis de documentales interactivos realizados en Colombia se plantean modelos que utilizan algunos recursos del arte digital y multimedia para su elaboración.

Hernández (2017) en su investigación Diseñando una recepción participativa para universos transmedia: roles y desafíos, presenta el papel activo que entran a desempeñar los públicos objetivos de las estrategias de difusión al evolucionar de consumidores a prosumidores de los contenidos. En Prácticas de la cultura digital y difusión del patrimonio. Videomapping y remix, de Lafluf y Barber (2017) se exponen obsevaciones y recomendaciones a partir del análisis de dos estudios de caso en el que la comunicación se ve mediada por la aplicación de tecnologías de última generación.

Las TIC aportan una serie de ventajas que hay que tener en cuenta a la hora de analizar el tipo de herramientas que se implementan para la elaboración de discursos en torno al patrimonio universitario:

- Su atractivo incorpora en los usuarios la motivación, que se genera a partir de la aceptación de estas herramientas, su intaractividad y la capacidad de presentar información de forma novedosa.

- La presentación de los contenidos es más detallada y de mayor calidad gráfica y sonora.

- La posibilidad de incorporar información procedente de diversas disciplinas, otorga a los discursos un carácter multidiscplinar.

Sin embargo la fuerza y rapidez con que se extiende el fenómeno globalizador constituye un peligro evidente y sin precedentes para la identidad cultural de 
las casa de altos estudios. En consecuencia para que realmente las TIC sean canales potentes al servicio de la comunicación global como refieren Mateos y Colorado (2008), siempre deben someterse a los principios de rigor y veracidad. Hay que tener en cuenta las posibilidades que ofrecen, para el diálogo, la comprensión y la cooperación. De esta forma "[...] en el diseño de la secuencia de procedimientos para la sensibilización, la estructura procedimental es: conocer, comprender, respetar, valorar, disfrutar y transmitir" (Fontal, 2003, p. 209).

\section{Conclusiones}

La comunicación de los valores del patrimonio universitario debe formar parte del concepto mismo de su gestión integral, desde el objetivo de la socialización y la creación de vínculos de integración cultural sobre la base del conocimiento, el reconocimiento y la difusión. $Y$ naturalmente no se puede pensar que la importancia que hoy se da a la comunicación es casual. Se basa en la idea de que el patrimonio adquiere todo su valor mediante la contemplación y comprensión por parte de los integrantes de la comunidad universitaria.

De esta forma se evidencia la necesidad de la aproximación entre las agendas de comunicación y de patrimonio, las cuales permitan contar con plataformas eficientes, que doten de carácter y personalidad propios al patrimonio universitario sobre la base de su identificación y representación. Así el dominio de sus límites, particularidades y alcance, facilitará propuestas de conexión entre los discursos y la visibilización interna y externa, sustentadas en la experiencia y las vivencias del ayer y el presente con visión de futuro. 


\section{Bibliografía}

- Arciniegas, A.T (2016). El documental interactivo como instrumento educativo. Propuesta teórico práctica para la difusión del patrimonio cultural colombiano. (Tesis Doctoral). Universidad Politécnica de Valencia. https://riunet.upv.es/handle/10251/76732

- Aznar, F. (2006). Educación artística, cultura y patrimonio. Educación artística y visual ante el reto social, cultura y territorialidad. Sevilla: Ed. COLBAA.

- Ballart, J. y Tresserras, J. (2001). Gestión del patrimonio cultural. Barcelona: Ariel.

- Castells, M. (2009). Comunicación y poder. España: Alianza.

- Campillo, R. (1998). La gestión y el gestor del patrimonio cultural. Murcia: Editorial KR.

- Capriotti, P. (2008). La planificación estratégica de la comunicación del patrimonio cultural. En S.M. Rusillo (Ed.). La comunicación global del patrimonio cultural (pp. 133-154). España: Gijón, Trea.

- Cristofano, M. y Palazzetti, C. (2011). II Museo verso una nuova identitá. Musei e comunitá, strategie comunicative e pratiche educative. [El Museo virtual una nueva identidad. Museo y comunidad, estrategia comunicativa y práctica educativa. Roma: Gangemi Editore.

- Consejo de Europa (2005). Recomendtion. [Recomendación]. Disponible en:

https://wcd.coe.int/ViewDoc.jsp?Ref=Rec\%282005\%2913\&Language=la nEnglish .

- Convención para la protección del patrimonio mundial, cultural y natural, (1972). Recuperado de: http://whc.unesco.org/en/35/ 
- Convención para la salvaguarda del patrimoniol cultural inmaterial, (2003). Recuperado de: http://www.unesco.org/culture/ich/es/convención

- Cutlip, S. M., Center, A. H., and Broom, G. M. (1994). Effective public relation. [Relaciones públicas efectivas]. Englewood Cliffs, Nueva Jersey: Prentice- Hall.

- Dávila, M., González , G. y Preciado, M. (2018). La radio como medio de comunicación del patrimonio cultural. Fernandina Radio: un estudio de caso. Universidad y Sociedad, 10(5), 416-423. Recuperado de: https://rus.ucf.edu.cu/index.php/rus/article/view/1081/1139

- Declaración de Budapest (2002). Recuperado de: http://portal.unesco.org/culture/es/files/29876/12651903953813BA79A.p df/813BA79A.pdf

- Díaz, G. (2008). Aspectos de la comunicación visual y gráfica en la comunicación del patrimonio cultural. En S.M . Mateos Rusillo (Coord.). La comunicación global del patrimonio cultural (pp. 175-270). España: Gijón, Trea.

- Escalante, E. A. y Huiltrón, L.A.(2017). Patrimonio arqueológico digital. Uso de las tecnologías de la información y la comunicación para la divulgación del patrimonio arqueológico. Conservación y Restauración, (n 11/12). INAH. Recuperado de: https://ceam.academia.edu

- Fagundes, D.(2010). Comunicación, cultura y sociedad digital en Brasil. Revista de Economía Política de las tecnologías de la Información y Comunicación. https://seer.ufs.br/index.php/eptic/article/view/62/36

- Fontal, O. (2003). La Educación Patrimonial. Teoría y práctica en el aula,el museo e internet. España: Ediciones Trea . 
- Fontal , O. (2008). La importancia de la dimensión humana en didáctica del patrimonio. En S.M. Mateos Rusillo (Ed.), La comunicación global del patrimonio cultural (pp.79-110). España: Gijón, Trea.

- García, M. P.(2011). El patrimonio cultural : conceptos básicos. Zaragoza: Prensas Universitarias de Zaragoza. Recuperado de: http://puz.unizar.es

- García,N. (1999). Los usos sociales del patrimonio cultural. En E. Aguilar, (Coord.), Patrimonio etnológico: nuevas perspectivas de estudio en Andalucía. España: Junta de Andalucía.

- García, N. (2006). Paranoicos versus utilitaristas. Revista semestral de cultura (35), pp. 5-6. Cantalunya: Nexus. Fundación Caixa

- Gómez, R. (2006). El plan de comunicación global de las organizaciones culturales. En R. Gómez de la Iglesia, La comunicación en la gestión cultural (pp.27-38). Vitoria-Gasteiz, País Vasco: Grupo Xabide.

- Hernández, J. (2017). Diseñando una recepción participativa para universos transmedia: roles y desafíos. Tropelías: Revista de Teoría de la Literatura y Literatura Comparada. https://dialnet.unirioja.es/servlet/articulo?codigo $=6081806$

- Hermon,S. y Noccolucci, F. (2007). Communicating Cultural Heritagein the 21st Century. The Chiron Project and its Research Opportunities. [Comunicando el Patrimonio Cultural en el siglo XXI. El Proyecto de Chiron y sus oportunidades de investigación]. Recuperado de: http://www.epochnet.org/index.php?option=com content\&task=view\&id= 202\&ltemid $=308$

- ICOMOS (2008). Carta para la Intertpretación y Presentación de Sitios del Patrimonio Cultural. Recuperado de: https://www.icomos.org. 
- Lafluf,M. y Barber, G.(2017). Prácticas de la cultura digital y difusión del patrimonio. Videomaping y remix. Encuentro latinoamericano de bibliotecarios, archivistas y museólogos: Revalorizando el patrimonio en la era

digital http://www.institutomora.edu.mx/EBAM/2017/Ponencias/Practicas\%20de \%20la\%20cultura\%20digital\%20y\%20difusion\%20del\%20patrimonio\%20 Videomapping\%20y\%20remix.pdf

- López, J.I (2000). Manual urgente para radialistas apasionados. La Habana: Editorial Pablo de la Torriente.

- Maiso, L; Safia,T. and Roland, W.(2013). Shaping European university heritage past and possible future.[Conformando el pasado de la universidad europea y su posible futuro]. Noruega, The Royal Norwegian Society and Akademika Publishing.

- Martín, M.J y Cuenca, J.M (2015). Educomuniación del patrimonio. https://www.researchgate.net/publication/277006981

- Martínez,J.C.(2016). Retos y soluciones en el empleo de las tecnologías 3D para la divulgación del trono de jaguar de Chichén Itzá. En D. Jiménez y M.Gándara (Coords).El patrimonio cultural y las Tecnologías Digitales. Experiencias recientes desde México. Instituto Nacional de Antropología e Historia, Consejo Nacional para la Ciencia y la Tecnología (pp.168-181).

- Mateos, S. M. (2008). Hacia una comunicación global del patrimonio cultural, o cómo potenciar su uso fomentando su preservación. En S.M. Mateos Rusillo (Coord.). La comunicación global del patrimonio cultural (pp.19-50). España: Gijón, Trea. 
- Mateos , S. M., y Colorado, A. (2008). Viejas y nuevas discusiones entre patrimonio artístico y cultural digital entre patrimonio artístico y cultural digital. Charla con Arturo Colorado. Miradas desde la copa. Revista de Comunicación y Patrimonio Cultural (1) pp-2-11.

- Mateos, S.M. (2009). El patrimonio cultural. En S. M. Mateos Rusillo (Coord.) Comunicación del patrimonio cultural (pp. 5-38). Barcelona: UOC.

http://www.comunicacionpatrimonio.net/wpcontent/uploads/2009/05/mira das 01.pf

- Mateos, S.M. (2012). Manual de comunicación para museos y atractivos patrimoniales.

- Mejías,J.(2008). Estructuras y principios de gestión del patrimonio cultural municipal. España: Ediciones Trea, Gijón.

- Mendes, S. (2002). Valores, patrimonio edificado y ciberespacio. Los usos del patrimonio cultural en la Sociedad de la Información. Recuperado de: http://unesdoc.unesco.org/images/0012/001284/128485s.pdf

- Morales, J. (2008). El sentido y metodología de la interpretación del patrimonio. En: S.M. Rusillo,(Coord.) La comunicación global del patrimonio cultural (pp.53-75). España: Ediciones Trea, Gijón.

- Niglio,O.(2013). Reflexiones sobre el valor del patrimonio cultural.En Mónica Aguilar Bonilla y Olimpia Niglio (Coords). La conservación del patrimonio cultural en Costa Rica (pp.23-38). Recuperado de: https//:www.academia.edu 
- Querol, M.A. y Martínez, B. (1998). Paso a paso: el tratamiento de los bienes arquelógicos en las leyes de patrimonio de Valencia y Madrid. Complutum (9) pp. 279-291. Madrid.

- Resick, M. (2017). Medios para comunicar el patrimonio: entramados comunicativos en la rehabilitación integral de Centro Histórico Habanaero. (Tesis de doctorado). Universidad de Granada.Recuperado de: $\underline{\text { http://hdll.handle.net/10481/48790 }}$

- Rivera, J.(2013). Declaración de Alcalá sobre la protección, conservación y difusión del patrimonio universitario. Madrid: Universidad de Alcalá de Henares

- Ruiz, D.(2011). Realidad aumentada y Patrimonio Cultural: nuevas perspectivas para el conocimiento y la difusión del objeto cultural. http://www.revistadepatrimonio.es/revistas/numero8/difusion/estudios2/p df/difusion-estudios2.pdf

- Talas, S. and Laurenço, M. (2012). Arranging and rearranging: planning university heritage for the future. [Organizar y reoganizar la planificación del patrimonio universitario para el futuro]. Padua, Italia: Padova University Press.

- Valdés, M. C. (1999). La difusión cultural en los museos: servicios destinados al gran público. España: EdicionesTrea, Gijón. 